\title{
Barriers to effective research supervision in clinical specialist training: Experience from a medical school in Malaysia
}

\author{
Yew Kong Lee, Chirk Jenn Ng, Joong Hiong Sim, Firdaus Amira, Chan Choong Foong, Wei \\ Han Hong, Junedah Sanusi, Adrian Jia Hwa Lim, Christopher Chiong Meng Boey
}

Lee YK, Ng CJ, Sim JH, et al. Barriers to effective research supervision in clinical specialist training: Experience from a medical school in Malaysia. Malays Fam Physician. 2021;16(3);77-86. https://doi.org/10.51866/oa1222

Keywords:

Supervision, research,

graduate medical education

\section{Authors:}

Lee Yew Kong

(Corresponding author)

$\mathrm{BA}, \mathrm{PhD}$

Department of Primary Care

Medicine, Faculty of Medicine

University of Malaya, Kuala Lumpur

Malaysia

Email: leeyk@um.edu.my

\section{$\mathrm{Ng}$ Chirk Jenn}

MBBS, MMed(Family Medicine), PhD

Department of Primary Care

Medicine, Faculty of Medicine

University of Malaya, Kuala Lumpur

Malaysia

\section{Sim Joong Hiong}

BSc (Hons), LLB (Hons), MEd, PhD

Medical Education and Research

Development Unit (MERDU), Faculty

of Medicine, University of Malaya

Kuala Lumpur, Malaysia.

Firdaus Amira

BSc, MA, PhD

Department of Media Studies, Faculty

of Arts \& Social Sciences, University

of Malaya, Kuala Lumpur, Malaysia

\section{Foong Chan Choong}

BScEd, PhD

Medical Education and Research

Development Unit (MERDU), Faculty

of Medicine, University of Malaya

Kuala Lumpur, Malaysia.

\begin{abstract}
Introduction: A compulsory research component is becoming increasingly common for clinical residents. However, integrating research into a busy clinical training schedule can be challenging. This study aimed to explore barriers to research supervision in specialist training programmes from the perspectives of clinical supervisors and trainees at a Malaysian university hospital.

Methods: Qualitative interviews and focus group discussions were conducted (December 2016 to July 2017) with clinical supervisors $(n=11)$ and clinical trainees $(n=26)$ utilising a topic guide exploring institutional guidelines, research culture and supervisor-student roles. Interviews were transcribed verbatim and analysed thematically to identify barriers to research supervision.

Results: Supervisors and trainees from 11 out of 18 departments participated. Both clinical supervisors and trainees struggled to successfully integrate a compulsory research component into residency training. Among the reasons identified included a lack of supervisory access due to the nature of clinical rotations and placements, clashing training priorities (clinical vs research) that discouraged trainees and supervisors from engaging in research, poor research expertise and experience among clinical supervisors hampering high-quality supervision, and a frustrating lack of clear standards between the various parties involved in research guidance and examination.

Conclusion: Both clinical supervisors and trainees struggled to successfully integrate a compulsory research component into residency training. This was not only an issue of resource limitation since questions regarding clinical priorities and unclear research standards emerged. Thus, institutional coordinators need to set clear standards and provide adequate training to make research meaningful and achievable for busy clinical supervisors and trainees.
\end{abstract}

\section{Introduction}

A mandatory research component is becoming increasingly common in medical specialist training. However, numerous barriers to research exist in specialist training, including insufficient time to conduct research, ${ }^{1,2}$ low interest ${ }^{3}$ and inadequate research skills. ${ }^{4}$

This study examines specialist training within an Asian context in Malaysia. Clinical specialist training programmes in Malaysia were established in the 1960s as master's level courses in public universities. ${ }^{5}$ Due to the master's accreditation, clinical trainees are required to conduct research as part of Malaysian master's standards criteria. ${ }^{6}$
Previous studies have reported that specialist trainees find research difficult and approach it reluctantly. ${ }^{1,2}$ From a pedagogical perspective, these trainees are now re-entering (postgraduate) medical training as adult learners-for whom engagement with a topic is essential to motivation. ${ }^{7,8}$ Therefore, if trainees are more motivated to become 'clinician-specialists' instead of 'clinicianscholars', the mandatory research component might fail to fulfil the learning needs of these adult learners.Studying the Malaysian context will be useful for discipline planning to establish more formal research standards. ${ }^{9}$ This study aimed to explore the barriers to research supervision perceived by research supervisors and postgraduate clinical trainees in a multi-disciplinary teaching hospital. 


\author{
Hong Wei Han \\ $\mathrm{PhD}$ \\ Medical Education and Research \\ Development Unit (MERDU), Faculty \\ of Medicine, University of Malaya \\ Kuala Lumpur, Malaysia.
}

\section{Junedah Sanusi}

$\mathrm{PhD}$

International Institute of Public Policy

\& Management (Inpuma), University

of Malaya, Kuala Lumpur, Malaysia

\section{Adrian Lim Jia Hwa \\ MBBS \\ Department of Primary Care \\ Medicine, Faculty of Medicine \\ University of Malaya, Kuala Lumpur \\ Malaysia}

Christopher Boey Chiong Meng $M B B S, D C H, M D, P h D, F R C P C H$, FRCP

Department of Paediatrics, Faculty of Medicine, University of Malaya Kuala Lumpur, Malaysia

\section{Methods}

Research design

Since little was known about this topic within a non-Western context, we used a qualitative design employing an interpretivedescriptive approach to explore the topic of research supervision. ${ }^{10}$ We obtained the views and perspectives of supervisors and trainees through in-depth interviews (IDIs) and focus group discussions (FGDs), which were then interpreted and described using thematic analysis. IDIs were used for clinical supervisors since in-depth interviews are suited to expert participants who can provide an overview of the topic. FGDs were chosen for the trainees to capitalise on their shared experiences and be triggered to discuss their research experience in greater detail.

\section{Setting}

This study was conducted in the Faculty of Medicine at the University of Malaya. This public university has the highest number of clinical specialist training programmes, ${ }^{5}$ with 18 clinical departments conducting 27 4-year clinical masters' programmes. ${ }^{11}$ These programmes can either be fully on-site at the university hospital or off-site/on-site with trainees spending the first 2 years of clinical training in public hospitals before returning to the university hospital. Trainees are required to complete a research project and submit a dissertation to obtain their master's degree. Dissertations are marked within the respective departments.

\section{Sampling and recruitment}

We used purposive sampling to recruit participants from each clinical programme. A total of 11 supervisors and 26 trainees agreed to participate in the study. For the supervisors, we interviewed the respective departmental postgraduate coordinators or lecturers who had experience in supervising research.

For clinical trainees, we only included candidates who had already started their research project (usually in the third or fourth year).

Data collection

The interviews and FGDs were conducted with interview guides based on Soren's domains of research supervision framework, ${ }^{12}$ which focuses on institutional guidelines, research culture, functional supervision (i.e., research mentorship), and student-supervisor roles. For supervisors, supervisory training and experience were also explored. The guides are provided in Appendices 1 and 2.

We conducted data collection between December 2016 and September 2017. The sessions were audio-recorded with a note taker present.

\section{Data analysis}

We used a thematic approach to data analysis. The audio recordings were transcribed verbatim. Next, five research team members (YKL, JHS, CCF, WHH and AJHL) jointly coded a transcript line-by-line and the codes were then collapsed to form larger categories. This formed an initial coding tree. Subsequently, two researchers (YKL and AJHL) used the coding tree to code the remaining transcripts. Any discrepancies in the coding were resolved by discussion until consensus was reached. The codes were discussed at two research team meetings. Having multiple members check and discuss the data helped to increase trustworthiness and avoid bias from a single perspective. The team members were a mix of clinicians and education researchers comprising a health psychologist, a primary care medicine specialist, a faculty-level postgraduate coordinator, an academic development researcher and medical education researchers. Data analysis was conducted iteratively, with data collection continuing until no new information was gathered or data saturation was reached.

\section{Ethics}

This study received ethics approval from the University of Malaya Medical Centre Medical Ethics Committee (Reference: MECID.NO: 20166-2530).

\section{Results}

Overall, 11 out of the 18 clinical departments participated in the present study. A total of 11 lecturer IDIs and 7 clinical trainee sessions ( $\mathrm{n}=5$ FGDs, $\mathrm{n}=2$ IDIs) were conducted. Participant demographics are reported in Table 1. 
Table 1. Participant demographic information.

\begin{tabular}{l|c} 
LECTURERS & $\mathbf{n = 1 1}$ \\
\hline Female & 7 \\
Male & 4 \\
\hline Age (range in years) & $40-47$ \\
\hline Completed a postgraduate research degree (e.g., PhD, DPhil) & 3 \\
\hline Postgraduate coordinator experience (range in years) & $1-6$ \\
\hline Position & 10 \\
Department clinical postgraduate coordinator & 1 \\
Coordinator for postgraduate research & $\mathbf{n = 2 6}$ \\
\hline POSTGRADUATE CLINICAL TRAINEES & 15 \\
\hline Female & 11 \\
Male & $29-38$ \\
\hline Age (range in years) & 4 \\
\hline Pursuing an academic medical career (i.e., sponsored by a public university) & $1-5$ \\
\hline Time between starting training and beginning research* (range in years) & $1-5$ \\
\hline Number of research supervisors per trainee (range) & 4 \\
\hline Clinical and research supervisors are different people & \\
\hline
\end{tabular}

* Defined as the calendar year in which the respondent began their research project after starting their master's programme.

Four main themes emerged as barriers to research supervision in clinical master's programmes: i) Access to research supervision; ii) Training priorities (clinical vs research); iii) Research expertise; iv) Varying research standards.

\section{Limited access to research supervision}

Poor accessibility to research supervision proved to be a significant barrier, especially for offsite/on-site programmes where trainees were away from the university for the first 2 years. Although this off-site placement was important for the development of clinical skills, both trainees and supervisors said that the distance between supervisor and trainee made it difficult for trainees to receive training and guidance on research since their contact time was limited.

I think they (the trainees) are busier outside. Their clinics are heavier. We can get some allocated time for classes and things like that. But I think in KKM (Ministry of Health settings), maybe not so much'. Trainee (FGD/CT_1)

'For those off-campus, our programme is 4 years; 2 years out, 2 years in. So, for the 2 years out, it is probably hard to communicate with the oncampus supervisors. Clinical supervisor (IDI/ CL_1)

Some programmes allowed students to start their research projects under their off-site supervisor. However, these projects often required corrections after students had returned to the university. Thus, some departments required students to present their research proposals shortly after their return to campus in order to monitor (and correct) these projects.

'We (trainees) have an off-campus supervisor and an on-campus supervisor. So, my research is conducted in my off-campus centre at $S_{-} g$ Hospital. Basically, I get most of my info from my off-campus supervisor, not so much from my oncampus supervisor, because my research is being done off-campus'. Trainee (FGD/CT_2_1)

'Some of them (trainees) have been reined back because they have not touched base with their academic supervisor. They have done their own thing in the Ministry of Health and we then found that actually it was not up to standard'.

Clinical supervisor (IDI/CL_2)

Training priorities (clinical vs research)

Participants pointed out that the primary purpose of the clinical masters training programme is to graduate trained specialists. Some respondents said that research was never given any prominence during earlier undergraduate or house officer training. Unsurprisingly, trainees said that they did not see the relevance of the research component. In courses where there were parallel specialist qualification routes (e.g., taking external fellowship examinations), trainees preferred those routes over the master's programmes since there was no compulsory research component.

'If you are destined to be a doctor, research is never a part of it. It is only a part of it when you enter a specialisation. When you do your housemanship, you are not really encouraged to do it... So, it is all about clinical, clinical, clinical work. Clinical supervisor (IDI/CL_4) 
'Many medical officers that would join the MRCP (Member of the Royal College of Physicians) and they would not need to produce a thesis. And you know, that is the benefit so to say for them. The benefit is obviously that you have less to work on... and some people will say, you know, the research component in the masters is a threat in itself'. Clinical supervisor (IDI/CL_5)

Some clinical coordinators echoed that research was not a priority. One felt that the research component could impair the trainees' clinical exam preparations and that research should not distract them from the exams. Some were frustrated at having to supervise research since they preferred clinical training and did not like research.

I have to say, my concentration is mainly on producing good clinical doctors... I'm not producing a scientist; I'm producing a clinician with an open mind'. Clinical supervisor (IDI/CL_5)

I'm a clinician, not a researcher. For me, the only reason I'm doing it, to be frank, is because I'm at the university. The university requires you to do it, so I do it. That's all. My main work is doing clinical work. I think if you ask 10 clinicians, they will tell you the same thing. Clinical supervisor (IDI/CL_3)

\section{A lack of research expertise}

Clinical lecturers are not required to possess a postgraduate research degree (e.g., $\mathrm{PhD}$ or MD). Thus, they may not be skilled or confident enough to supervise postgraduate research. To overcome this, research supervision in some departments was delegated to more 'research-oriented' lecturers.

'But for research, they (trainees) can take anybody. So, there is no specific research supervisor... Some (lecturers) are very clinically inclined, and they themselves don't do that much research, so they do not supervise. So, these are the ones who do not get any students. So, those who are more research-oriented will get more students. Clinical supervisor (IDI/CL_7)

For off-site programmes, participants noted that hospital-based clinical supervisors did not give much attention to research. Another issue was a mismatch between trainees' and supervisors' research areas and interests. This resulted in off-site trainees initiating projects based on their off-site supervisor's interests, which then had to be continued with their onsite supervisors.
'KKM (Ministry of Health) is not so happy because we (the University) greatly emphasise so much on research. They said most of them did not do research anyway'. Clinical supervisor (IDI/ CL_7)

'My topic is usually based on my external or offcampus supervisor. So, it's a bit difficult because my topic is a bit more relevant to my off-campus supervisor. So, my on-campus supervisor cannot contribute as much as my off-campus supervisor. So, most of the time I will go to my off-campus supervisor'. Trainee (FGD/CT_2_1)

\section{Varying standards of research}

In general, departments could be divided into two types according to their research standards: easy (where most-if not all—of the trainees pass the research component) and difficult (where trainees were strictly examined and failed if they did not meet the examination standards). In the former, standards for research were generally minimal, and trainees who demonstrated a general understanding of the research process would pass. In the latter, trainees became disgruntled due to varying standards since this significantly affected their likelihood of passing. They pointed out three types of discrepancies that were faced: i) between supervisors (e.g., some expected more complex methodologies); ii) between supervisors and examiners (e.g., failure due to a discrepancy between supervisors' and examiners' standards); iii) between supervisors and research experts (e.g., receiving different advice when consulting statisticians).

'Sometimes, during the (research) presentation, I feel like it's like a closed circuit; you and your supervisor in one (circuit) and now (during the presentation) it is open. Communication is not [occurring] between other examiners or lecturers, or between them and the statistician. [It] is like you are alone. [It] is like [the rest of them] totally do not communicate sometimes, I feel. Like, some say the statistics [should be] like this but then the lecturer says, "No, you have to interpret it this way". But they never meet [all] three together. You are just stuck in between'. Trainee (FGD/ CT_3_1)

'Different lecturers have different opinions... some of the tests, like the reliability test - a simple thing like this - some say you don't have to do it. You know, different opinions. So, there is no proper guide and I think there is no standard on what to do'. Trainee (FGD/CT_3_2) 
Varying standards led to confusion and frustration among trainees who expected their supervisors to be able to guide them. Without good early guidance, it could be too late for students to raise questions after the data collection had been performed. Although trainees were not worried about passing or failing in departments where minimal standards were employed, external examiners noted that the research fell short of their standards.

'Actually, there was one candidate who didn't count the sample size. So, I was wondering if, let's say, you know, he did it wrong from the beginning, why didn't the supervisor say anything about it?' Trainee (CT_3_2)

'There were times we found out that we have an oral progress presentation where all the faculty will be present. That's when you are asked, "How come you didn't do this step and we do this step?" And you can't say, "I've been told by my supervisor not to do that step"' Trainee (CT_3_4)

'I think one of the things is that, uh, now they try to initiate things (research support) to be more structured because there were remarks from the past external examiner that our student theses were not-I mean for them - up to their expectation. Trainee (IDI/CT_4)

\section{Discussion And Conclusion}

This study shed light on how contextual issues played an important role in the emergence of barriers to research. The issues of research access, priority, expertise and standards found in this study are likely found in most clinical specialist training programmes. ${ }^{1,2,13}$ Thus, discussions on these issues are relevant to programmes elsewhere.

The participants in our study highlighted the lack of access to research supervision, especially when off-site. Barriers to access have been raised in other hospital-based research settings, where trainees recognise that being in the same location is key to having more supervisor access. ${ }^{14}$ Thus, in settings where students and supervisors are in different locations, it is important to strategise about how trainees can best utilise 'off-site' time for research. For example, universities can look into forming collaborations with hospital clinical research centres, where research-trained staff could provide students with on-site research support. Moreover, clinical (i.e., hospitalbased) supervisors could also contribute to research by generating researchable areas from their clinical setting and experience. However, academic supervisors may need to strike a balance between academic research, healthsystem-based research and clinical audit. Furthermore, off-site trainees and academic supervisors should leverage the use of online video-conferencing platforms to increase access and meeting frequency. This will also help to reduce the difference in the amount of teaching received on research techniques between off-site and on-site trainees.

The question about training priorities (i.e., the priority and place of research in clinical master's training) was pertinent to our study. As mentioned in the introduction, Malaysia's inclusion of compulsory research in specialist training programmes was a master's standards decision made 40 years ago. This contrasts with the more recent inclusion of research in other countries, which generally aims to develop clinician-scholars who advance the field. ${ }^{16}$ However, trainees apparently fail to link the importance of compulsory research with their experience as medical practitioners. Engagement in learning only occurs when adults know why they need to learn new things (i.e., research), which might be exacerbated when the learning is against their internal motivation (i.e., compulsory). ${ }^{7}$ Understanding the context of how views are formed is the first step. The next step involves re-framing clinicians' views of research in health systems like Malaysia's, where capacity building for service delivery is the dominant narrative and policy thrust. In this context, service orientation could be leveraged by reminding trainees and supervisors that conducting research feeds into a positive cycle of clinical skills improvement. Academic role models who actively translate research results into practice are important in this regard.

Another concern was the lack of research expertise available in the clinical setting. Others have also reported a paucity of experienced researchers in specialist training centres due to a historical lack of hiring or producing clinical staff with research experience. ${ }^{1}$ If formal standards for supervisors are eventually introduced, there will be an insufficient number of qualified supervisors, which would lead to the unequal distribution of supervisory responsibilities. Thus, policies and strategies must be put in place by institutions to train existing clinicians for research supervision along with the tandem requirements for research skills (e.g., implementing structured research 
skill programmes for lecturers and providing training opportunities for postgraduate research degrees) and supervisory skills (e.g., supervising research projects, advising on when or how to seek help, co-supervision and knowing the minimum standards required for a student to pass their research component). With skilled lecturers in place, adequate preparation should be provided for students before embarking on their research project through multi-faceted development programmes that can include training workshops, facilitated lecturer access and project presentation meetings. ${ }^{17}$ In recent years, more training workshops have specifically targeted clinical trainees. To further facilitate this process, train-the-trainer workshops should be conducted to enhance the skills of lecturers in guiding their trainees.

The last issue was that of setting standards for research in clinical programmes. Rothberg et al. (2014) observed that the requirements for scholarly activity in US graduate medical programmes have been purposively left vague to allow each programme to fulfil the requirements in their own way. ${ }^{18}$ However, our study shows that there are training contexts in which research standards need to be clearer. If research forms part of the accreditation for clinical specialist training (e.g., as in Malaysia, Singapore and South Africa), a clear set of standards would be beneficial and should outline whether or not the standards of research in a clinical master's are equal to those of a master of science degree. ${ }^{1,19}$ In the last few years, major reviews of the national postgraduate medical training curriculum have been conducted by most clinical specialities. These have involved looking at the standards required for the research component of clinical training programmes. Another context in which clear standards are required includes clinician-scholar programmes such as the academic clinical fellowship programmes in the United Kingdom, where doctors interested in pursuing careers in academic medicine gain both a clinical specialist qualification and a $\mathrm{PhD} .{ }^{20}$ If exposure to scholarly activity is the goal of the research component, a full-blown research project is unnecessary and participation in an ongoing research project would suffice. ${ }^{18}$ Another model would be to provide residents with protected research time via an additional year for research. Notably, this has been shown to double publication output in a 5- vs 6-year residency program. ${ }^{21}$ Thus, the goal of research in a clinical training programme must be clear before standards can be set accordingly.

This study had several limitations that might have affected the study findings. For example, not all departments were represented in this study since some chose not to participate. Thus, there may be barriers or facilitators to research supervision that were not captured.

Research supervision within clinical training programmes can be frustrating for both trainees and supervisors. This struggle is not just an issue of time or resource limitation since questions about clinical priorities and unclear research standards emerged in this study. Identifying and addressing these contextual issues is important to ensure that trainees can engage in meaningful research within clinical training programmes.

\section{Acknowledgements}

We wish to thank all of the participants who took part in the study. We also thank the Faculty of Medicine, University of Malaya for funding the study.

\section{Conflicts of interest}

There are no conflicts of interest to declare. 


\section{References}

1. Aldous C, Clarke D, van Wyk J, et al. Avoiding the distant elephant: A model to approach the research component of specialization. $B M C$ Med Educ. 2016;16:147.

2. Kabra SK, Verma IC. Thesis during MD: Must or bust? Indian J Pediatr. 2007;74:868-869.

3. Silcox LC, Ashbury TL, VanDenKerkhof EG, et al. Residents' and program directors attitudes toward research during anesthesiology training: A Canadian perspective. Anesth Analg. 2006;102:859-864.

4. Bammeke F, Liddy C, Hogel M, et al. Family medicine residents' barriers to conducting scholarly work. Can Fam Physician. 2015;61:780-87.

5. Lim V. Postgraduate Medical Education and Training. Newsletter of the Academy of Medicine Malaysia. 2010;19.

6. Malaysian Qualification Agency. Standards: Master's and Doctoral Degree. Petaling Jaya: Malaysian Qualification Agency; 2015.

7. Knowles MS, Holton E, Swanson R. The Adult Learner: The Definitive Classic in Adult Education and Human Resource Development. Burlington: Elsevier; 2005.

8. Taylor DC, Hamdy H. Adult learning theories: Implications for learning and teaching in medical education: AMEE Guide No. 83 Med Teach. 2013; 35:e1561-72.
9. Simpson D, Meurer L, Braza D. Meeting the scholarly project requirement-Application of scholarship criteria beyond research. J Grad Med Educ. 2012;4:111-2.

10. Thorne S. Interpretive Description. Walnut Creek: Left Coast Press, 2008.

11. Faculty of Medicine, University of Malaya. Postgraduate Programmes Offered by the Faculty of Medicine 2017 [Internet] Available from: https://medicine.um.edu.my/ postgraduate\#section2. Accessed 16 September 2017.

12. Bengsten SSE. Review of the Handbook Literature on Doctoral Supervision. Denmark: Centre for Teaching Development and Digital Media, Aarhus University; 2014.

13. Peyton CC, Badlani GH. Dedicated research time in urology residency: Current status. Urology. 2014;83:719-24.

14. Caldwell PH, Oldmeadow W, Jones CA Supervisory needs of research doctoral students in a university teaching hospital setting. J Paediatr Child Health. 2012;48:907-12.

15. Ministry of Health Malaysia. Clinical Research Centre 2017 [Internet]. Available from: http:// www.crc.gov.my/. Accessed 16 September 2017.
16. Ahmad S, De Oliveira GS, Jr., McCarthy RJ. Status of anesthesiology resident research education in the United States: Structured education programs increase resident research productivity. Anesth Analg. 2013;116:205-10.

17. Carter AE, Anderson TS, Rodriguez KL, et al. A program to support scholarship during internal medicine residency training: Impact on academic productivity and resident experiences. Teach Learn Med. 2019;31:552-65.

18. Rothberg MB, Kleppel R, Friderici JL, et al. Implementing a resident research program to overcome barriers to resident research. Acad Med. 2014;89:1133-9.

19. Chew $\mathrm{CH}$, Chee YC. Postgraduate medical education and specialist training in Singapore. Ann Acad Med Singapore. 2005;34:182c-189c.

20. Funston G, Cerra C, Kirkham D, et al. The road to a clinical academic career. BMJ Careers [Internet]. Available from: http://careers.bmj. com/careers/advice/The_road_to_a_clinical_ academic_career. Accessed 16 September 2017.

21. Finkelstein JB, Van Batavia JP, Rosoff JS. The difference a year can make: Academic productivity of residents in 5 vs 6-Year urology programs. Urology. 2015;86:220-2. 


\section{APPENDIX}

\section{APPENDIX 1: Topic Guide for Postgraduate Clinical Supervisors}

\section{Introduction}

1. How long have you been supervising the research component of clinical master's students?

2. How many students are you supervising now?

3. How has your experience of supervision been?

We would like to ask you about your practice of research supervision.

\section{Institutional}

1. How do you usually supervise your students (research skills?)? Does your department have a set of guidelines on research supervision (e.g. frequency of meetings, supervisory forms, supervision contracts?)

a. Do you co-supervise? If yes, how do you decide the supervisory roles?

2. Is there anything that hinders your supervision?
a. Probe - time constraints/multiple roles
b. Probe - too many rules and regulations
c. Probe - lack of support

\section{Research culture/ Critical thinking}

1. What else do you teach your students besides research skills to help them to be better researchers?
a. Ethics
b. Grant application processes
c. Dissemination (conferences)
d. Scientific communication (e.g. language for grant writing, conferences)

2. What is your expectation of the student's research?
a. Goal of a research component (pass the student? publication?)
b. How does this affect your supervision?
c. How do you deal with conflict with the student?
i. What is the source of these conflicts?

\section{Functional supervision}

1. Do you teach them research skills i.e. skills needed to plan, conduct and write up a thesis (one-to-one, workshops)? If yes, how?

2. How do you monitor the progress of your student?

a. How do you give feedback to your students? Do you ask for feedback from your students?

3. How do you motivate your students to do research?

a. Barriers to conducting research (emotional, disinterested, personal problems, stressed over doing research)

4. How do you prepare your students for their research/thesis exams?

a. Do you explain to the students what the thesis examination format is like?

5. What if your students fail their research exams? How do you support them? 


\section{Student-Supervisor Roles and Relationship}

1. Does your relationship with the student change over time? Could you describe your relationship with your student?

2. Do you approach clinical and research supervision differently? If yes, how?

a. Do you switch roles between clinical supervision and research supervision? If yes, how?

b. Do you blend research and clinical supervision (e.g. clinical justification, recommendations for future research) when supervising the same student?

c. What would you say is your priority? Why do you say so?

i. Do you think a research component is necessary for a clinical master's program?

3. How do you feel about research supervision?

a. Probe: Enjoyable or not? Meaningful or not? And why?

b. How does your experience of research supervision compare to clinical supervision?

4. What benefits do you get out of this research supervision?

a. Probe: Publication, producing good researchers/ future academicians.

Training for research supervisors

1. Are there any criteria for becoming a research supervisor in your department?

2. Have you gone for any training to become a research supervisor?

3. What support do you need as supervisors?
a. Support for supervisors
b. Support for students

\section{APPENDIX 2: Topic Guide for Postgraduate Clinical Trainees}

\section{Introduction}

1. How long have you worked on the clinical master's research component?

2. How many supervisors do you have now?

3. How has your experience of supervision been?

We would like to ask you about your experience of research supervision.

\section{Institutional}

3. Can you tell me how your supervision for the research component of your masters has been?? Does your department have a set of guidelines on research supervision (e.g. frequency of meetings, supervisory forms, supervision contracts?)

a. Are you co-supervised? If yes, how do your supervisors work with you?

4. Is there anything at your department that hinders your masters research?
a. Probe - structure of masters programme
b. Probe - too many rules and regulations/unclear rules and regulations
c. Probe - Lack of support

\section{Research culture/ Critical thinking}

3. What else has your research supervisor taught you besides research skills to help you to become a better researcher?
a. Ethics
b. Grant application processes
c. Dissemination (conferences)
d. Scientific communication (e.g. language for grant writing, conferences) 
4. What do you think is the expectation for the research that you are doing?

a. Goal of a research component (pass the thesis exam? publication?)

b. How does this affect how you approach the research component?

c. Have you experienced any conflict with your supervisor?

i. If yes, what is the source/s of these conflicts?

\section{Functional supervision}

6. Did your supervisor teach you research skills i.e. skills needed to plan, conduct and write up a thesis

a. How did he teach you? i.e. one-to-one, workshops

7. Does your supervisor monitor your research progress?

a. How does he/she you give you feedback on your progress? Do you ask for feedback from your supervisor?

8. How does your supervisor motivate you to do research?

a. What are your barriers to conducting research (emotional, disinterested, personal problems, stressed over doing research)? How does your supervisor help you with these?

9. Does your supervisor prepare you for your research/thesis exams?

a. Do you know what the thesis examination format is like?

10. (For students beyond Year 4) Have you failed your research exams? How did your supervisor respond?

\section{Student-Supervisor Roles and Relationship}

5. Does your relationship with your supervisor change over time? Could you describe your relationship with your supervisor?

6. Do you think of clinical and research supervision differently? If yes, how?

d. Do you switch roles between being a clinical supervisee and research student? If yes, how?

e. Do you blend research and clinical supervision (e.g. clinical justification, recommendations for future research)?

f. What would you say is your priority? Why do you say so?

i. Do you think a research component is necessary for a clinical master's program?

7. How do you feel about research supervision?

a. Probe: Enjoyable or not? Meaningful or not? And why?

b. How does your experience of research supervision compare to clinical supervision?

8. What benefits do you get out of this research supervision?

a. Probe: Publication, becoming good researchers/ future academicians.

\section{Training for research supervisors}

4. Are you aware of any criteria or training for appointing research supervisors in your department?

5. What support do you need for your research component?

a. Support from supervisors

b. Support for students 